\title{
Randomized Trials of Frequent Hemodialysis - Infinite Possibilities
}

\author{
Rita S. Suri ${ }^{a}$ Amit X. Garg ${ }^{a, b}$ \\ a Division of Nephrology, London Health Sciences Center, and ${ }^{b}$ Department of Epidemiology and Biostatistics, \\ University of Western Ontario, London, Ont., Canada
}

\section{Key Words}

Frequent hemodialysis · End-stage renal disease . Dialysis regimens

\begin{abstract}
Current understanding of the benefits of daily and nocturnal hemodialysis is limited by the designs used in previous studies which tested these therapies. There is increasing momentum to evaluate these therapies in randomized controlled trials. By establishing the efficacy, safety and delivery of daily and nocturnal hemodialysis, such trials will guide policy and funding decisions regarding the role of these therapies in the treatment of end-stage renal disease. However, in designing trials that fulfill these goals, many questions arise. What study population should be used? How should the intervention be defined? Which outcomes should be evaluated? Several methodological issues particular to trials of frequent dialysis require additional consideration. The 'ideal' trial in terms of methods must be balanced against the feasibility of implementation and budgetary constraints. Here, we explore some of these issues.
\end{abstract}

Copyright @ 2006 S. Karger AG, Basel

\section{Introduction}

The prevalence of end-stage renal disease (ESRD) is increasing, the cost of renal replacement therapy is high, and the disease and treatment are associated with a significant loss in patients' quality of life [1-3]. Three times weekly hemodialysis (HD) remains the major treatment modality for ESRD. Despite advances in dialysis technologies and general medical care, the $20 \%$ annual mortality rate of dialysis patients has not decreased in the last decade [4]. Increasing the delivered dialysis dose in conventional HD does not appear to improve mortality or other clinical outcomes as shown in a recent randomized trial $[5,6]$.

Since 1998, more frequent HD regimens have been gaining attention worldwide. Typically delivered as either short daily (1.5-3 h/session, 6 days/week) or long nocturnal (6-8 h/session, 6 days/week) treatments, frequent HD provides significantly increased weekly small solute, phosphate, and water clearances compared with conventional HD (3-4 h/session, 3 days/week) [7, 8]. These treatments may be performed either in-center or at home, allowing patients increased flexibility. Several observational studies have suggested that both daily and nocturnal HD may result in improved health and quality of life for patients with ESRD [9-14]. Unfortunately, findings from these studies have been variable. In addi-

\section{KARGER}

Fax +4161306 1234 E-Mail karger@karger.ch www.karger.com 
tion, the majority of these studies suffer from several methodological limitations, including small sample size, use of non-ideal control groups, and drop-out and selection biases $[15,16]$. Thus, many believe that randomized trials are now needed to clarify the true benefits of frequent HD. These trials should not only establish the efficacy and safety of daily and nocturnal HD, but should also serve to guide governments and funding agencies in making policy decisions regarding the role of these potentially expensive therapies in the treatment of ESRD. The trials should also inform nephrologists on how these new therapies may best be implemented in real practice.

In designing trials that fulfill these goals, many questions arise. There are potentially endless possibilities with respect to the choice of the study population, intervention, control group, outcomes, and economic evaluation. Several methodological issues particular to trials of frequent dialysis require additional consideration. The 'ideal' trial in terms of methods must be balanced against budgetary constraints and feasibility of implementation and recruitment. In the following discussion, we explore some of these issues.

\section{Study Population}

One major limitation of previous studies of frequent HD is that participants were often not representative of the general HD population. Whether highly select participants differ from the general HD population in their response to these therapies is unclear. For example, in studies of daily HD, the mean age of study participants was 45-64 years, their mean time on conventional HD had been $2-11$ years, $0-28 \%$ had diabetes, and almost half of the patients were selected because they were 'stable' or able to undergo HD at home [16]. Participants enrolled in past nocturnal studies had similar characteristics, with a mean age of 40-50 years, and less than one third having diabetes [10, 13, 17].

The study population chosen for any trial should reflect the population for whom the therapy is intended [18]. This raises the following questions: for whom is frequent HD intended? Should all patients with ESRD be offered daily or nocturnal HD? If not, what are the eligibility criteria? Obviously, the answers to these questions depend on the type and location of the therapy. As mentioned, previous studies suggested that frequent HD is currently being prescribed to patients who are younger and healthier than the general HD population $[10,13$, $16,17]$. However, some have suggested that in-center dai- ly HD should be preferentially used in very ill patients who require extra clearance or ultrafiltration, including those with severe malnutrition, uncontrolled hypertension, recurrent pulmonary edema, or frequent intradialytic hypotension $[11,12]$. Similarly, nocturnal HD may be targeted to those with severe soft tissue and vascular calcification where high phosphate clearances are required [19]. On the other hand, when performed at home, only the most stable, compliant, and motivated patients with adequate social support may be eligible and interested in these therapies [20]. Cost considerations may also preclude those with a poor life expectancy from undergoing home daily or nocturnal HD as a result of high initial capital investments required for home HD [21].

Once inclusion criteria for any trial are decided, it should be appreciated that all eligible patients may not be interested in participating, potentially causing selection biases [22]. The increased cost and inconvenience of traveling to the HD unit twice as often may deter patients from considering in-center daily HD; moreover, daily transportation may not be available even for those who are willing. Similarly, many patients are reluctant to undergo home nocturnal HD due to fears about performing the procedure themselves, lack of social support, or an aversion to 'medicalize' their home [23]. Alternatively, some patients are simply unwilling to participate in a clinical trial. Patients who decline participation for these reasons may be less motivated, of lower socioeconomic status, or more ill than those who agree to participate. These differences could potentially affect their response to therapy, limiting the generalizability of the trial's results [24].

Thus, in conducting a trial of frequent HD, the collection of baseline demographic data and the reason for nonparticipation of patients who are excluded from the trial should be considered. These data could not only aid trial recruitment efforts but could guide assessments of generalizability and improve the understanding of how to best apply the therapy outside of the trial. For example, if the major reason for non-acceptance of in-center daily HD is increased transportation costs, supplemental funding would help to remove this barrier. Similarly, supplementing capital costs for home modifications or developing increased home care support may improve the acceptance of home nocturnal HD in those with financial barriers or with poor social support, respectively. If it is found that participants are younger and healthier than eligible non-participants, it may be impractical to expect that frequent HD will be applied to the majority of the general HD population. Unfortunately, the collection of 
detailed data on non-participants who have not consented to the trial may be limited by federal privacy legislation.

\section{Study Intervention}

\section{Time, Frequency, and Dose}

In the last 3 decades, several frequent and intensive dialysis modalities have been employed, including long conventional (6-8 h/session, 3 days/week) [25], enhanced conventional (3-4 h/session, 4 days/week) [26], short daily (2-3 h/session, 5-7 days/week) [16], and long nocturnal (6-8 h/session, 5-7 days/week) [15]. Each of these regimens has been reported to have beneficial outcomes in observational studies compared with conventional HD, and thus may be a potential option for study in a randomized trial.

It is not clear whether the intervention should simply be based on time and frequency, or whether a dialysis 'dose' should be specified. If the dose is specified, how should it be measured? Several methods exist to estimate weekly urea clearance [27-29], but none have been rigorously validated. However, the frequent HD intervention is defined, ideally, there should be a clear separation between study and control groups in the clearance of small and middle molecules, phosphate, and water. Such separation will likely be relatively easy to achieve with any long nocturnal HD regimen, even if performed for only 6 h, 5 nights/week (Gotch/Greene, unpubl. data). However, the ability to achieve such high clearances with short daily HD may be limited by patient willingness to accept long treatment times 6 days/week (Gotch, unpubl. data). As a result, the daily HD intervention may need to be defined by the highest dose that patients will tolerate.

\section{Location and Independence}

The intervention chosen for a trial of frequent HD must consider location and independence of care in addition to time, frequency, and dose. Although nocturnal HD is typically performed independently at home by the patient and/or caregiver, other modalities may be performed either in-center or at home with varied levels of independence, ranging from total nursing care, nurse-assisted self-care, to complete independence. The choice of location and independence of care will affect the generalizability of the trial results, as patients able to undergo home independent HD are typically more compliant, motivated, and healthier than patients who must dialyze incenter [20]. More importantly, the location and type of care chosen will affect how the study results are interpreted, depending on the control group. In a trial of home nocturnal HD, if the control group receives conventional HD at home, then any differences in outcomes observed will most likely be related to increased dialysis dose. However, if the control group receives conventional HD incenter, then being at home becomes an intricate part of the intervention, and observed effects attributed to increased dialysis dose will be potentially confounded by effects related to being at home. The impact of this confounding will depend on the outcomes chosen: quality of life outcomes will likely be more affected than objective physiological measures. Ultimately, the choice of either option depends on the goals of the study, as well as the feasibility of implementation. If the standard of care is conventional in-center HD, then studying the double intervention of 'home' and 'nocturnal' against the standard of care may be the most feasible option. On the other hand, given that home nocturnal HD is likely to be considerably more expensive than home conventional HD [30], some would argue that these two interventions should be studied separately in order to properly evaluate the effects and cost effectiveness of each.

\section{Non-Dialytic Aspects of the Intervention}

Just as being at home may become part of the intervention in a trial of home nocturnal HD, the intervention of in-center daily HD is complex and involves aspects of care unrelated to dialysis dose. Patients on in-center daily HD come to the HD unit twice as often as those on conventional HD, with increased opportunities for interaction with the health-care team and fellow patients. Patients receiving in-center short daily HD are assessed daily by nurses and may interact with nephrologists, physiotherapists, nutritionists, pharmaceutical and social workers more frequently than patients on conventional HD. Compliance may also improve for those medications given on dialysis. In the setting of a positive trial result, it may be argued that any quality of life or physiological benefits conferred by short daily HD may relate to increased surveillance and care by the medical team rather than increased HD dose. As it is impractical to completely eliminate this source of confounding, any increased time spent with the health-care team as a result of coming to the HD unit daily becomes an inherent aspect of the intervention. Thus, in order to guide the interpretation of the trial results, efforts should be made to characterize the non-dialytic aspects of the frequent intervention. This would include gathering data on the number of visits and time spent with the health-care team in each arm. 


\section{Outcomes and Economic Evaluation}

\section{Primary Outcome}

The primary outcome chosen for any trial of frequent HD should fulfill several requirements. Obviously, the intervention should be hypothesized to have a clinically important effect on the outcome. However, the outcome should also have substantial importance to patients, practitioners, and funding agencies, so that a positive trial will elicit change in policy as well as in practice. This latter aim must be weighed against the feasibility of recruiting the necessary sample size and monies available to fund the trial. Thus, although a trial demonstrating improved mortality with frequent HD may be ideal in influencing practice, the required sample size of thousands of patients with years of follow-up would likely preclude its current undertaking. Not only does the complexity of the intervention make the costs to conduct such a large trial prohibitive, it is unclear whether such large numbers of patients would be willing to participate. Moreover, embarking on such a large trial would be imprudent, without first obtaining more experience in multiple centers with this novel therapy that has, to date, been utilized by few.

For these reasons, the evaluation of 'softer' endpoints will likely be the most feasible current option. Previous studies have suggested that frequent HD improves a multitude of health parameters, including health-related quality of life [31], blood pressure [32, 33], left-ventricular mass index [34, 35], hemoglobin [36], albumin [37], dietary protein intake [37], and phosphate [38]. Although improvements in all or several of these outcomes may be compelling enough to establish the efficacy of frequent $\mathrm{HD}$, it is unclear which of these outcomes should be chosen as the primary outcome of a trial designed with the objective of affecting policy. Health-related quality of life, although clinically important, would likely be inadequate on its own given it is a self-reported measure potentially subject to placebo effects in an unblinded trial. Of the other surrogate physiological measures, few have been validated against a change in mortality for this patient population. Thus, it is likely that any trial of frequent HD will require the evaluation of multiple outcomes.

\section{Evaluation of Safety}

Currently, the safety of frequent HD is uncertain. With increased dialysis, there is the potential for increased blood loss, more vascular access events, watersoluble vitamin deficiency, as well as patient burnout and increased burden on caregivers. It is imperative that trials evaluate each of these outcomes, so that physicians and their patients can consider the risk-benefit when using these therapies.

\section{Cost-Effectiveness}

If daily and nocturnal HD are shown to improve outcomes, then implementation of frequent HD as an alternative treatment option for patients with ESRD will depend on its associated costs. While home daily and nocturnal HD cost less than in-center conventional HD [30, 39], this is not the case for in-center daily HD. Given the cost-effectiveness and cost utility of any of these therapies are largely unknown, evaluating these outcomes in a trial of frequent HD would have important implications. The detail with which the economic evaluation is conducted and the breadth of its perspective will depend on resources available for necessary data collection, and the interest of those conducting the trial. Funding agencies may be most interested in costs from the perspective of the health care provider. However, understanding the costs borne by patients and caregivers is equally important. Such costs may be related to transportation, home dialysis modifications, or lost time from work to attend HD training sessions. Even if frequent HD is demonstrated to be cost-effective from the viewpoint of the funding agency, these therapies are unlikely to be adopted into practice if costs borne by the patient and caregiver are deemed excessive.

\section{Conclusion}

Randomized trials of frequent HD are now required. These trials should be designed to meet the objectives of establishing the efficacy and safety of frequent HD, guiding funding agencies in making policy decisions, as well as ascertaining how such therapies may best be implemented outside the trial. Whether or not the future trials of frequent HD influence policies and practices remains to be seen. 


\section{References}

1 Merkus MP, Jager KJ, Dekker FW, Boeschoten EW, Stevens P, Krediet RT: Quality of life in patients on chronic dialysis: self-assessment 3 months after the start of treatment. The Necosad Study Group. Am J Kidney Dis 1997; 29:584-592.

-2 Valderrabano F, Jofre R, Lopez-Gomez JM: Quality of life in end-stage renal disease patients. Am J Kidney Dis 2001;38:443-464.

\3 Walters BA, Hays RD, Spritzer KL, Fridman M, Carter WB: Health-related quality of life, depressive symptoms, anemia, and malnutrition at hemodialysis initiation. Am J Kidney Dis 2002;40:1185-1194.

-4 Goodkin DA, Young EW, Kurokawa K, Prutz KG, Levin NW: Mortality among hemodialysis patients in Europe, Japan, and the United States: case-mix effects. Am J Kidney Dis 2004;44(suppl 3):16-21.

-5 Eknoyan G, Beck GJ, Cheung AK, Daugirdas JT, Greene T, Kusek JW, et al: Effect of dialysis dose and membrane flux in maintenance hemodialysis. N Engl J Med 2002;347:2010_ 2019.

-6 Unruh M, Benz R, Greene T, Yan G, Beddhu S, DeVita M, et al: Effects of hemodialysis dose and membrane flux on health-related quality of life in the HEMO study. Kidney Int 2004; 66:355-366.

7 Suri R, Depner TA, Blake PG, Heidenheim AP, Lindsay RM: Adequacy of quotidian hemodialysis. Am J Kidney Dis 2003;42(suppl 1):42-48.

8 Pierratos A: Effect of therapy time and frequency on effective solute removal. Semin Dial 2001;14:284-288.

-9 Andre MB, Rembold SM, Pereira CM, Lugon JR: Prospective evaluation of an in-center daily hemodialysis program: results of two years of treatment. Am J Nephrol 2002;22:473479.

10 Pierratos A, Ouwendyk M, Francoeur R, Vas S, Raj DS, Ecclestone AM, et al: Nocturnal hemodialysis: three-year experience. J Am Soc Nephrol 1998;9:859-868.

11 Traeger J, Galland R, Delawari E, Arkouche W, Hadden R: Six years' experience with short daily hemodialysis: do the early improvements persist in the mid and long term? Hemodial Int 2004;8:151-158.

-12 Ting GO, Kjellstrand C, Freitas T, Carrie BJ, Zarghamee S: Long-term study of high-comorbidity ESRD patients converted from conventional to short daily hemodialysis. Am J Kidney Dis 2003;42:1020-1035.
13 Lockridge RS, Spencer M, Craft V, Pipkin M, Campbell D, McPhatter L, et al: Nightly home hemodialysis: five and one-half years of experience in Lynchburg, Virginia. Hemodial Int 2004;8:61-69.

14 Lindsay RM: The London, Ontario, Daily/ Nocturnal Hemodialysis Study. Semin Dial 2004; 17:85-91.

15 Walsh M, Culleton B, Tonelli M, Manns B: A systematic review of the effect of nocturnal hemodialysis on blood pressure, left ventricular hypertrophy, anemia, mineral metabolism, and health-related quality of life. Kidney Int 2005;67:1500-1508.

16 Suri RS, Nesrallah GE, Mainra R, Garg AX, Lindsay RM, Greene T, et al: Daily hemodialysis: a systematic review. Clin J Am Soc Nephrol 2006, in press.

17 Lindsay RM, Leitch R, Heidenheim AP, Kortas C: The London Daily/Nocturnal Hemodialysis Study - Study design, morbidity, and mortality results. Am J Kidney Dis 2003; 42(suppl 1):5-12.

18 Guyatt G, Cook D, Devereaux PJ, Meade M, Strauss S: Therapy; in Guyatt G, Rennie D (eds): Users' Guide to the Medical Literature. Chicago, AMA Press, 2002, pp 55-79.

19 Kim SJ, Goldstein M, Szabo T, Pierratos A: Resolution of massive uremic tumoral calcinosis with daily nocturnal home hemodialysis. Am J Kidney Dis 2003;41:E12.

20 Mowatt G, Vale L, MacLeod A: Systematic review of the effectiveness of home versus hospital or satellite unit hemodialysis for people with end-stage renal failure. Int $\mathbf{J}$ Technol Assess Health Care 2004;20:258-268.

21 Kroeker A, White S, Lindsay R: Return on investment: an economic guideline for selecting home daily/nocturnal hemodialysis patients (abstract). Hemodial Int 2004;8:97.

22 Bjarnason NH, Kampmann JP: Selection bias introduced by the informed consent process. Lancet 2003;361:1990.

23 McLaughlin K, Manns B, Mortis G, Hons R, Taub K: Why patients with ESRD do not select self-care dialysis as a treatment option. Am J Kidney Dis 2003;41:380-385.

24 Dans A, McAlister F, Dans L, Richardson WS, Strauss S, Guyatt G: Therapy and applying the results: applying results to individual patients; in Guyatt G, Rennie D (eds): Users' Guide to the Medical Literature. Chicago, AMA Press, 2002, pp 369-384.

25 Charra B, Jean G, Chazot C, Hurot JM, Terrat JC, Vanel T, et al: Intensive dialysis and blood pressure control: a review. Hemodial Int 2004; 8:51-60.

26 Twardowski Z: Effect of long-term increase in the frequency and/or prolongation of dialysis duration on certain clinical manifestations and results of laboratory investigations in patients with chronic renal failure. Hemodial Int 2004; 8:30-38.
27 Gotch FA: The current place of urea kinetic modelling with respect to different dialysis modalities. Nephrol Dial Transplant 1998;13(suppl 6):10-14

28 Casino FG, Lopez T: The equivalent renal urea clearance: a new parameter to assess dialysis dose. Nephrol Dial Transplant 1996;11:15741581 .

29 Keshaviah P: The solute removal index - A unified basis for comparing disparate therapies. Perit Dial Int 1995;15:101-104.

30 McFarlane PA: Reducing hemodialysis costs: conventional and quotidian home hemodialysis in Canada. Semin Dial 2004; 17:118-124.

31 Heidenheim AP, Muirhead N, Moist L, Lindsay RM: Patient quality of life on quotidian hemodialysis. Am J Kidney Dis 2003; 42(suppl 1):36-41.

32 Koshikawa S, Akizawa T, Saito A, Kurokawa $\mathrm{K}$ : Clinical effect of short daily in-center hemodialysis. Nephron Clin Pract 2003;95:c23c30.

-33 Nesrallah G, Suri R, Moist L, Kortas C, Lindsay RM: Volume control and blood pressure management in patients undergoing quotidian hemodialysis. Am J Kidney Dis 2003;42(suppl 1):13-17.

34 Chan CT, Floras JS, Miller JA, Richardson RM, Pierratos A: Regression of left ventricular hypertrophy after conversion to nocturnal hemodialysis. Kidney Int 2002;61:2235-2239.

35 Fagugli RM, Reboldi G, Quintaliani G, Pasini P, Ciao G, Cicconi B, et al: Short daily hemodialysis: blood pressure control and left ventricular mass reduction in hypertensive hemodialysis patients. Am J Kidney Dis 2001;38: 371-376.

36 Piccoli GB, Mezza E, Quaglia M, Bermond F, Bechis F, Burdese M, et al: Flexibility as an implementation strategy for a daily dialysis program. J Nephrol 2003; 16:365-372.

37 Galland R, Traeger J: Short daily hemodialysis and nutritional status in patients with chronic renal failure. Semin Dial 2004; 17:104-108.

38 Mucsi I, Hercz G, Uldall R, Ouwendyk M, Francoeur R, Pierratos A: Control of serum phosphate without any phosphate binders in patients treated with nocturnal hemodialysis. Kidney Int 1998;53:1399-1404.

39 Kroeker A, Clark WF, Heidenheim AP, Kuenzig L, Leitch R, Meyette M, et al: An operating cost comparison between conventional and home quotidian hemodialysis. Am J Kidney Dis 2003;42(suppl 1):49-55. 